\title{
Prédiction du débit sédimentaire à l'aide de la formule de Dibajnia et Watanabe
}

\author{
Paulo Alves da Silva*, André Temperville*** \\ *Enseignant, Département de Physique, Université d'Aveiro, 3810 \\ Aveiro, Portugal. \\ **Professeur Emérite, L.E.G.I., B.P.53, 38041 Grenoble cedex 9, \\ France
}

Résumé

La formulation du débit sédimentaire sous l'action combinée des houles et des courants est un problème important pour les modèles hydrosédimentaires qui n'est pas encore bien maîtrisé. Dans le cas d'une houle linéaire, du second ordre, ou cnoidale en interaction avec un courant, nous proposons une formule paramétrée à l'aide des valeurs du courant, de la vitesse quadratique moyenne et du coefficient d’asymétrie de la houle en se basant sur la formule de base de Dibajnia et Watanabe. Après quelques modifications de la formule de base, les résultats obtenus semblent en bon accord avec une centaine d'expériences.

Abstract

The sand transport by the interaction of waves and current is an important problem linked to coastal morphodynamic process and an accurate forecast is sometimes very difficult. Based on the Dibajnia and Watanabe model, we give a new formula for the sediment transport for linear wave, second order wave or cnoidal wave, in interaction with a current. The formula parameters are the current amplitude, the root mean square of the wave velocity and the asymmetry coefficient of the wave. The results are in good accordance with experiments.

\section{Introduction}

La prévision de l'évolution des fonds marins et de l'érosion des côtes est un défit important pour les années à venir. Ceci est lié d'une part à l'impact des interventions humaines de plus en plus nombreuses sur les zones côtières dans un but soit économique soit touristique et d'autre part à l'élévation prévue du niveau moyen des mers en relation avec les variations climatiques.

Une modélisation correct du transport sédimentaire en zone côtière nécessite une bonne représentation de l'hydrodynamique résultant de l'interaction des houles et des courants et en particulier une bonne paramétrisation de la couche limite de fond et de la turbulence qui y est générée. Les nombreux travaux ont été réalisés ces vingt dernières années dans ce domaine: Grant et Madsen (1979), 
Fredsoe (1984), Davies et al. (1988), Huynh Thanh et Temperville (1990), Myrhaug et Slaattelid (1990). Ces travaux sont analysés dans les divers ouvrages de base: Sleath (1984), Horikawa (1988), Fredsoe et Deigaard (1992), Nielsen (1992), Van Rijn (1993) et Soulsby (1997). Le cas des houles aléatoires a été abordé récemment par Guizien et Temperville (1999).

Le lien avec les processus de morphodynamique côtière est réalisé à travers les formules de transport (sable ou vase). Nous n'envisagerons ici que le cas du transport de sable. Ce transport a en général un caractère complexe tridimensionnel. On distingue: a)- le transport longshore qui est lié all courant de marée et/ou le courant induit parallèlement à la côte par la houle b)- le transport cross-shore qui est dominé par le mouvement orbital des houles et qui est beaucoup plus difficile à prévoir. De grosses lacunes existent encore dans la modélisation du transport de sédiments. C'est pourquoi plusieurs programmes de la CEE participent au développement d'outils opérationnels dans ce domaine. C'est le cas en particulier du projet SEDMOC (SEDiment transport MOdelling in marine Coastal environments) qui a pour objectif à la fois d'utiliser des modèles sophistiqués et aussi de proposer des formules à usage plus pratique comme dans la présent communication. Dans sa thèse, Dohmen-Janssen (1999) fait une analyse détaillée des processus entrant en jeu dans le transport sédimentaire sous un écoulement alternatif et montre l'influence important pour le débit net du retard de phase des valeurs temporelles de la concentration sur celles de la vitesse.

Dans les ouvrages cités précédemment, on peut trouver de nombreuses formules qui ont été proposées pour le transport sédimentaire sous l'action des houles et des courants. Nous ne retiendrons que la formule de Bailard (1981) qui relie le transport à la puissance instantanée de l'écoulement et généralise ainsi la formulation de Bagnold pour un écoulement constant et la formule de Dibajnia et Watanabe (que nous noterons par la suite D-W) qui sera étudiée en détail ici et qui prend en compte les interactions possible des deux alternances (positive et négative) de l'écoulement oscillant dues au déphasage entre les valeurs temporelles des concentrations et des vitesses.

Dans le paragraphe 2 nous rappellerons les principes de base de la formule de Dibajnia et Watanabe. Nous proposerons une formule paramétrée à partir de DW dans le paragraphe 3 et dans le paragraphe 4 nous comparerons avec différentes expériences qui nous amènerons à proposer quelques modifications.

\section{Le modèle de Dibajnia et Watanabe}

Considérons un profil de vitesse unidimensionnel connu u(t) de période $T$ (figure 1), composé de deux alternances de durées respectives $T_{1}$, où, dans la première la vitesse est positive $(i=c)$ et dans la deuxième la vitesse est négative $(i=t)$.

Ce modèle estime le transport total (charriage plus suspension) en faisant lat différence entre les transports pendant l'alternance positive et l'alternance négative. Pour ce faire on utilise un paramètre $\Gamma$ défini par: 


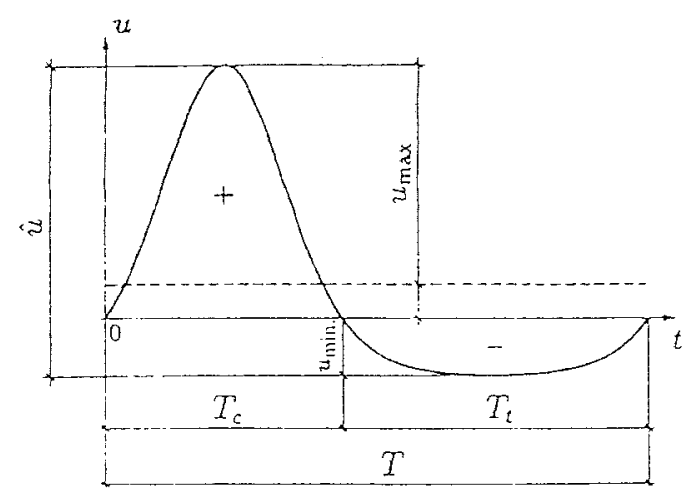

Fig. 1 - Série temporelle des vitesses, définitions Time series of velocity, definitions

$$
\Gamma=\frac{u_{c} T_{c}\left(\Omega_{c}{ }^{3}+\Omega_{t}{ }^{3}\right)-u_{t} T_{t}\left(\Omega_{t}{ }^{3}+\Omega_{c}{ }^{3}\right)}{T\left(u_{c}+u_{t}\right)}
$$

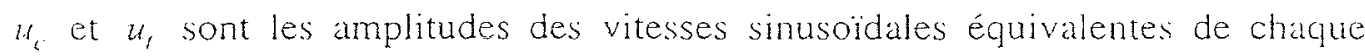
alternance $(i=c$ et $i=t), \Omega_{c}$ est un nombre adimensionnel proportionnel à la quantité de sable mise en suspension, transporté et déposée pendant l'alternance positive et $\Omega_{\prime}^{\prime}$ est un nombre adimensionnel proportionnel à la quantité de sable mise en suspension pendant l'alternance négative précédente qui n'a pas été déposée et qui est donc transportée pendant l'alternance positive. II en est de même pour le transport pendant l'alternance négative. Les échanges de sable entre les deux alternances dépendent de la valeur de $\omega_{i}$, qui est un nombre adimensionnel caractérisant le rapport entre le temps de chute des particules dont la vitesse de chute est $W$ et la période $T_{i}$ de l'alternance correspondante ( $i=c$ et $i=t)$ :

$$
\begin{aligned}
& u_{i}^{2}=\frac{2}{T_{i}} \int_{0}^{T_{i}} u^{2}(t) d t \quad, \quad \omega_{i}=\frac{u_{i}^{2}}{2(s-1) g W T_{i}} \\
& \text { si } \omega_{i} \leq \omega_{c r}\left\{\begin{array}{l}
\Omega_{i}=\frac{2 \omega_{i} W T_{i}}{d_{50}} \\
\Omega_{i}^{\prime}=0
\end{array} \text {, si } \omega_{i} \geq \omega_{c r}\left\{\begin{array}{l}
\Omega_{i}=\frac{2 \omega_{c r} H T_{i}}{d_{50}} \\
\Omega_{i}^{\prime}=\left(\bar{\omega}_{i}-\bar{\omega}_{c, r}\right) \frac{2 W T_{i}}{d_{50}}
\end{array}\right.\right.
\end{aligned}
$$

$g$ représente l'accélération de la gravité, $d_{5 n}$ représente le diamètre moyen du grain de sable et $s=\rho_{s} / \rho$, où $\rho_{s}$ et $\rho$ représente la densité du sédiment et de l'eau. Le valeur critique de $\Phi_{i}, \Phi_{c r}$, dépend du régime de transport (sheet flow. rides).

Le débit sédimentaire total, $q$, est évalué par la formule (Dibajnia et Watanabe, 1992):

$$
\frac{q}{W d_{50}}=\alpha \mid \Gamma^{\beta} \frac{\Gamma}{|\Gamma|}
$$


Dans la formule d'origine donné par Dibajnia et Watanabe (1992), qui avait été ajustée avec des expériences faites avec du sable de diamètre $0.2 \mathrm{~mm}$ et dans des conditions de sheet flow, les valeurs de $\alpha$ et de $\beta$ sont égales à 0.001 et 0.55 et $\omega_{c r}=1$. En 1995 Dibajnia a proposé une formule généralisée sous forme vectorielle et dont nous parlerons au paragraphe 3.1 .2 et donné une loi pour $\bar{\omega}_{c r}$ en fonction du paramètre de Shields, $\Theta=\frac{\tau_{b}}{(s-1) g d_{50}}\left(\tau_{b}\right.$ est le frottement au fond), pour prendre en compte le régime de rides:

$$
\begin{array}{ll}
\text { si } \Theta \leq 0.2 & \bar{\omega}_{C r}=0.03 \\
\text { si } 0.2 \leq \Theta \leq 0.6 & \bar{\omega}_{c r}=1-0.97 \sqrt{1-((\Theta-0.2) / 0.4)^{2}} \\
\text { si } 0.6 \leq \Theta & \bar{\omega}_{C r}=1
\end{array}
$$

Après une étude avec des sables de granulométrie étalée, Dibajnia et Watanabe (1996) proposa pour $\alpha$ et $\beta$ les valeurs 0.0015 et 0.5 et modifie l'expression du nombre adimensionnele de référence, $\Omega_{i}$ :

$$
\Omega_{i}=\bar{\omega}_{i} T_{i} \sqrt{\frac{(s-1) g}{d_{50}}}
$$

\section{Paramétrisation de la formule de Dibajnia et Watanabe.}

Pour une houle sinusoidale et pour des raisons de symétries, la formule de D-W donne un débit sédimentaire nul. Cependant dans la majorité des cas nous sommes en présence d'une hydrodynamique plus complexe avec des houles asymétriques (du type houle de Stokes du $2^{\text {ème }}$ ordre ou houle cnoiddale) souvent en présence d'un courant moyen colinéaire ou non avec une intensité un. C'est pourquoi non traiterons successivement les cas suivants:

- houle plus courant colinéaire

- houle de Stokes du $2^{\text {ème }}$ ordre

- houle cnoïdale

- courant plus houle oblique.

Le cas des houles aléatoires ne sera pas traité pour l'instant mais un commentaire sera fait en fin de paragraphe. Dans toute la suite nous utiliserons pour les houles les variables suivants: la moyenne quadratique de la vitesse, $\mathrm{u}_{r m s}$, et le coefficient d'asymétrie, $r$, définis par :

$$
u_{r m s}^{2}=\frac{1}{T} \int_{0}^{T} u^{2}(t) d t \quad, \quad r=\frac{u_{\max }-u_{\min }}{u_{\max }+u_{\min }}
$$

Le champ de vitesses correspond à celui qui existe juste à la limite supérieure de la couche limite du fond. 


\subsection{Cas d'un courant plus une houle colinéaire}

Si $u_{0}=r_{0} u_{r m s}$ caractérise le courant seul, le champ de vitesses est donné par :

$$
u(t)=u_{r m s}\left[r_{0}+f(t)\right]
$$

$f(t)$ est une fonction de l'asymétrie, $r$, et de la pulsation, $\omega$, de lat houle et,

$$
<f(t)>=0 \text { et }\left\langle f^{2}(t)>=1\right.
$$

$<>$ représente une moyenne temporelle. Le cas où $r_{0}=0$ représente la houle seule.

3.1.1. Houle de Stokes du $2^{\text {eme }}$ ordre.

Pour une houle de Stokes du $2^{\text {ime }}$ ordre, on a :

$$
f(t)=\frac{\sqrt{2}(\cos \omega t+r \cos 2 \omega t)}{\sqrt{1+r^{2}}}
$$

On définit deux nombres adimensionneles pour caractériser les temps $T_{c}$ et $T_{1}$. Ces deux nombres sont donnés par:

$$
\frac{T_{c}}{T}=t_{c} \quad \frac{T_{t}}{T}=1-t_{c}=t_{1}
$$

Alors, les valeurs de $u_{c}$ et $u_{f}$ sont données par:

$$
\begin{aligned}
& u_{c}=u_{r m s} \gamma_{c}, \quad \gamma_{c}^{2}=2\left(1+r_{0}^{2}+\frac{\gamma_{p}}{\pi t_{c} \sqrt{1+r^{2}}}\right) \\
& u_{t}=u_{r m s} \gamma_{r}, \quad \gamma_{t}^{2}=2\left(1+r_{0}^{2}-\frac{\gamma_{p}}{\pi\left(1-t_{c}\right) \sqrt{1+r^{2}}}\right) \\
& \gamma_{p}=\frac{\sin \pi t_{c}\left[13 r+19 r_{c}+X\left(18 r r_{c}+1\right)\right]}{6}, \text { avec }: r_{c}=\sqrt{\frac{1+r^{2}}{2} r_{0}} \\
& X=\cos \pi t=\frac{-1+\sqrt{1+8 r^{2}-8 r r_{c}}}{4 r}, \text { si } r \neq 0, \text { et }: X=-r_{c} \text { si } r=0 .
\end{aligned}
$$

\subsubsection{Houle cnoidale}


Le champs des vitesses d'une houle cnoïdal est donné par :

$$
u(t)=\frac{A\left[c n^{2}(t, k)-\overline{c n^{2}}\right]}{1-\overline{c n^{2}}} \quad \overline{c n^{2}}=1+\frac{1}{k^{2}}\left(\frac{E}{K}-1\right)
$$

où $c n(t, k)$ est la fonction cosinus elliptique de paramètre $k$ (qui peut varier de 0 à I et caractérise l'asymétrie). $\overline{c n^{2}}$ représente la valeur moyenne de $c n^{2}(t, k)$ et $E(k), K(k)$ sont les intégrales elliptiques complètes de $1^{\text {ière }}$ et $2^{\text {ième }}$ espèce. La période est donné par $T=2 K(k)$. Le coefficient d'asymétrie, $r$, est :

$$
r=\frac{1-\overline{c n^{2}}}{1+\overline{c n^{2}}}
$$

On sait (Byrd et Friedman, 1954) que $u(t)=\frac{1}{k^{2}} \frac{d Z(t)}{d t}$ où $Z(t)$ est la fonction de Jacobi qui est donné avec une bonne approximation par:

$$
Z(t) \approx \frac{\sin \omega t}{a-b \cos \omega t}
$$

où $a$ et $b$ sont des fonctions de $k$. Après quelques calculs et en introduisant $u t_{\text {mms }}$ et $r$, on déduit que :

$$
\begin{aligned}
& u(t)=\frac{\sqrt{2} u_{r m s}\left(1-r^{2}\right)(\cos \omega t-r)}{(1-r \cos \omega t)^{2}}, \text { et : } \\
& f(t)=\sqrt{2}\left(1-r^{2}\right)^{\frac{3}{4}} \frac{(\cos \omega t-r)}{(1-r \cos \omega t)^{2}}
\end{aligned}
$$

Les temps $T_{c}$ et $T$, et les valeurs de $u_{c}$ et $u_{\text {, sont obtenus par des formules }}$ semblables avec:

$$
\begin{aligned}
& u_{c}=u_{r m s} \gamma_{c}, \quad \gamma_{c}^{2}=2\left(r_{0}^{2}+\frac{\gamma_{p}}{\pi t_{c}}\right) \\
& u_{t}=u_{r m s} \gamma_{i} \quad, \quad \gamma_{1}^{2}=2\left(r_{0}^{2}-\frac{\pi-\gamma_{p}}{\pi\left(1-t_{c}\right)}\right) \\
& \left.\gamma_{p}=2 \mid f_{0}+\sin \pi t_{c}\left(\sqrt{2} r_{0} f_{1}+f_{2}\right)\right] \\
& f_{0}=\operatorname{Arctg}\left[\sqrt{\frac{1+r}{1-r}} \operatorname{tg}\left(\frac{\pi t_{c}}{2}\right)\right] \quad f_{1}=\frac{\left(1-r^{2}\right)^{\frac{3}{4}}}{1-r X}
\end{aligned}
$$




$$
\begin{aligned}
& f_{2}=\frac{\left(1-r^{2}\right)^{\frac{3}{2}}}{3 r}\left[\frac{1-r^{2}}{(1-r X)^{3}}-\frac{1}{2(1-r X)^{2}}-\frac{\left(4 r^{2}-1\right)}{2\left(1-r^{2}\right)(1-r X)}\right] \\
& X=\cos \pi r_{c}=\frac{2 r r_{c}-1+\sqrt{1+4 r_{c} r^{3}-4 r r_{c}}}{4 r^{2} r_{c}}, \text { si } r_{c} \neq 0, \text { et }: X=r \text {, si } r_{c}=0
\end{aligned}
$$

Ainsi connaissant $u_{m, m}, u_{0}$ ( ou $r_{0}$ ) et $r$, il est possible de calculer $T_{c}, T_{r}$, $u_{c}, u_{t}$ et le débit sédimentaire selon la méthode de D-W exposée dans le paragraphe précédent.

$r_{0}=0, r \neq 0$, correspond au cas d'une houle asymétrique sans courant; $r_{0} \neq 0, r=0$, au cas d'un courant plus une houle sinusoïdal et si $r^{r}=r_{0}=0$, on trouve que $T_{c}=T_{t}=T / 2, u_{c}=u_{1}$ et le débit sédimentaire est nul.

Dans la réalité, les houles sont irrégulières et sont donnés par la nature du spectre (par exemple, un spectre de Jonswap). Avec la série temporelle de la vitesse, il est possible de calculer $u_{r m s}$, et en appliquant les définitions du paragraphe 2 , les valeurs de $T_{c}, T_{t}, u_{c}$ et $u_{t}$, en fonction des caractéristiques du spectre (par exemple, $H_{s}$ et $T_{p}$ ). On peut alors calculer le débit sédimentaire selon le même principe.

\subsection{Cas d'une houle plus un courant oblique}

Les résultats précédents peuvent être généralisés au cas d'un courant oblique par rapport à la houle. Considérons une houle se propageant dans la direction $O x$ et un courant $u_{n}$ faisant un angle $\theta$ avec la houle. La vitesse instantanée est donné par:

$$
\vec{u}(t)=\vec{u}_{0}+u_{i} \cdot m s f(t) \vec{i}=\left(u_{0} \cos \theta+u_{r m s} f(t)\right) \vec{i}+u_{0} \sin \theta \vec{j}=u_{x}(t) \vec{i}+u_{y} \vec{j}(10)
$$

Nous définissions comme dans le cas colinéaire $T_{c}, T_{t}, u_{c x}$ et $u_{t x}$ pour la composant sur $O x$ de $\vec{u}(t)$. Il suffit de remplacer dans les formules précédentes $u_{0}$ par $u_{0} \cos \theta$. On a alors:

$$
u_{c}^{2}=\frac{2}{T_{c}} \int_{0}^{T_{c}} u^{2}(t) d t=u_{c x}^{2}+2 u_{()}^{2} \sin ^{2} \theta=u_{c x}^{2}+u_{c y}^{2}
$$

En posant $u_{c y}=\sqrt{2} u_{0} \sin \theta=\sqrt{2} u_{y}=u_{t y}$, on a $\vec{u}_{c}=u_{c x} \vec{i}+u_{c y} \vec{j}$ et de même $\vec{u}_{t}=u_{t x} \bar{i}+u_{t y} \vec{j}$.

Remarquons que le coefficient $\sqrt{2}$ est absent dans la formule proposée par Dibajnia (1996) ce qui nous semble être une erreur.

Le paramètre de transport est donné sous sa forme vectorielle:

$$
\bar{\Gamma}=\frac{T_{c} \vec{u}_{c}\left(\Omega_{c}^{3}+\Omega_{t}^{\prime 3}\right)+T_{t} \vec{u}_{t}\left(\Omega_{t}^{3}+\Omega_{c}^{\prime 3}\right)}{\left(\left|u_{c}\right|+\left|u_{t}\right|\right) T}=\Gamma_{x} \vec{i}+\Gamma_{y} \vec{j}, \text { avec : }
$$




$$
\Gamma_{x}=\frac{T_{c} u_{c x}\left(\Omega_{c}^{3}+\Omega_{t}^{\prime 3}\right)-T_{t} u_{t x}\left(\Omega_{t}^{3}+\Omega_{c}^{\prime 3}\right)}{\left(\left|u_{c}\right|+\left|u_{t}\right|\right) T}, \Gamma_{y}=\frac{T_{c} u_{c y}\left(\Omega_{c}^{3}+\Omega_{t}^{\prime 3}\right)+T_{t} u_{t y}\left(\Omega_{t}^{3}+\Omega_{c}^{\prime 3}\right)}{\left(\left|u_{c}\right|+\left|u_{t}\right|\right) T}
$$

Le débit sédimentaire est donné par :

$$
\frac{\vec{q}}{W d_{50}}=\alpha \mid \Gamma^{\beta} \frac{\bar{\Gamma}}{|\Gamma|}
$$

\section{Applications}

A l'aide des expressions analytiques précédentes, il est possible d'étudier la variation du débit en fonction des caractéristiques de l'écoulement : la valeur du courant moyen, le $u_{r m s}$ de la houle, son coefficient d'asymétrie et la période ainsi que le diamètre des grains de sable. Par exemple, sur la figure 2 nous avons représenté les isolignes du débit sédimentaire adimensionnel en fonction de $r$ et $r_{0}$ pour des valeurs fixées de $u_{r m s}, T$ et $d_{50}$ pour une houle de Stokes du $2^{\text {iène }}$ ordre $\left(u_{r m s}=67.5 \mathrm{~cm} / \mathrm{s}, T=4 \mathrm{~s}\right.$ el $\left.d_{s n}=0.2 \mathrm{~mm}\right)$.

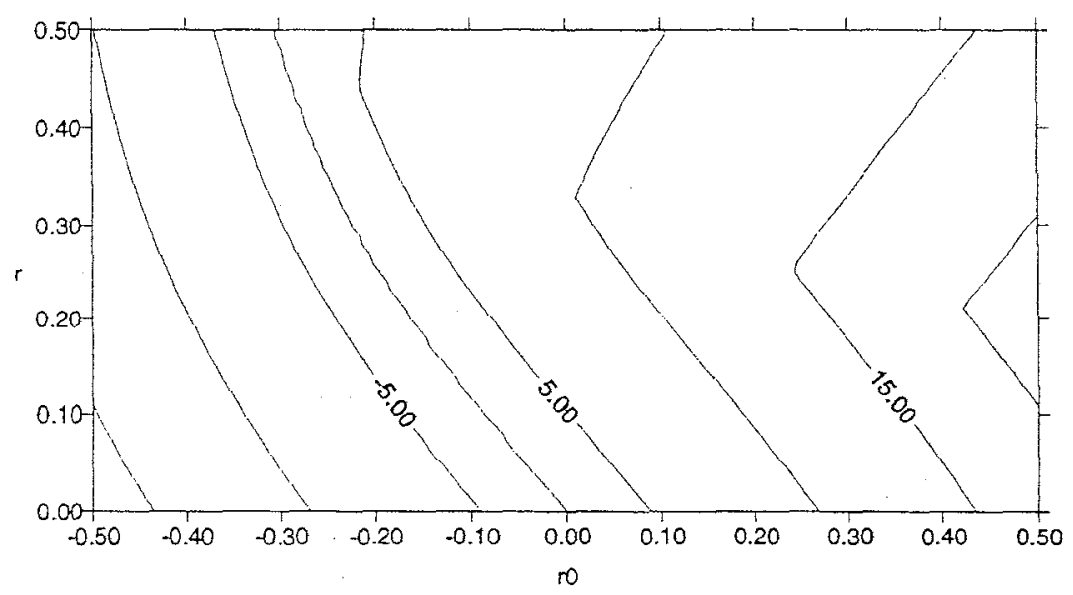

Fig. 2 - Isolignes du débit sédimentaire adimensionnel pour une houle de Stokes du $2^{\text {ieme }}$ ordre avec courant $\left(u_{r m s}=67.5 \mathrm{~cm} / \mathrm{s}, T=4\right.$ s et $\left.d_{50}=0.2 \mathrm{~mm}\right)$.

Isolines of dimensionless net transport rate for $2^{\text {nd }}$ order Stokes wave with current

Sur la figure 3 nous avons comparé les résultats obtenus par notre formulation avec les résultats expérimentaux de Janssen et Ribberink (1996) dans le cas d'une faible granulométrie. Il apparaît de façon évidente sur la courbe pointillée que les mécanismes d'interaction entre les deux alternances (positive et négative) ne sont pas bien pris en compte pour les faibles granulométries dans les conditions de sheet flow. Pour décrire correctement ces expériences, nous avons considéré que $\varpi_{c r}$ dépend du paramètre de Shields, $\Theta$, selon une loi qui à été ajustée avec les expériences et est donné ci-dessous. Les résultats qui apparaissent sur la ligne continue montrent une très bonne concordance avec les résultats expérimentaux. 


$$
\begin{array}{ll}
\text { Si } \Theta \leq 0.2 & \varpi_{c r}=0.03 \\
\text { Si } 0.2 \leq \Theta \leq 0.4 & \varpi_{c r}=1-\sqrt{1-((\Theta-0.2) / 0.58)^{2}} \\
\text { Si } 0.4 \leq \Theta \leq 1.5 & \varpi_{c r}=0.8104 \sqrt{\Theta}-0.4225 \\
\text { Si } 1.5 \leq \Theta & \varpi_{c r}=0.7236 \sqrt{\Theta}-0.3162
\end{array}
$$

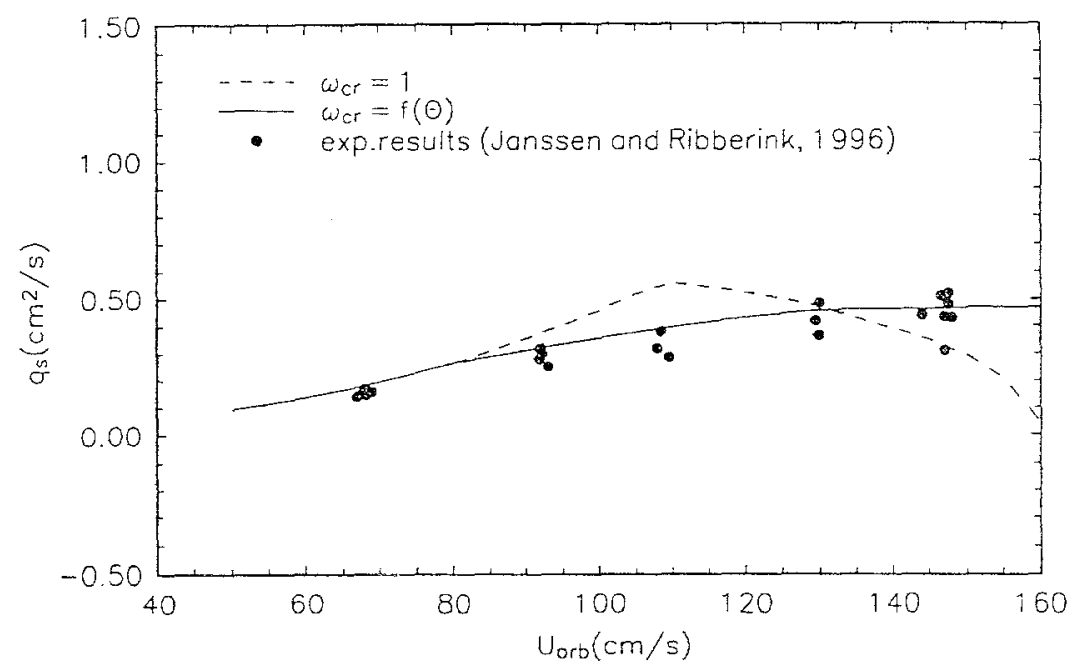

Fig. 3 - Comparaison du débit sédimentaire calculé et mesuré pour des cas d'interaction houle courant : $u_{0}=23 \mathrm{~cm} / \mathrm{s}, T=7.2 \mathrm{~s}, d_{50}=0.13 \mathrm{~mm}$ ). Comparison of the computed and measured net sediment transport rate for the combined wave-current case.

La nouvelle forme proposée pour $\bar{\omega}_{c r}$ a été testée avec la formulation de Dibajnia et Watanabe (1996) pour une centaine d'expériences de différents auteurs et pour différents granulométries (voir tableau 1). Les résultats qui sont présentés sur la figure 4 sont corrects dans une rapport 2 pour pius de $60 \%$ des cas (dans le cas du régime de sheet flow cette valeur est presque 100\%).

\begin{tabular}{|c|c|c|c|}
\cline { 2 - 4 } \multicolumn{1}{c|}{} & $\mathrm{d}_{50}(\mathrm{~mm})$ & régime & référence \\
\hline serie $\mathrm{E}$ & 0.21 & $\mathrm{sf}(\mathrm{O})$ & Katopodi et al. (1994) \\
serie B & 0.21 & $\mathrm{sf}(+\Delta) \mathrm{r}(\bullet)$ & Ribberink and Al-Salem(1994) \\
$\mathrm{CI}+\mathrm{CII}$ & 0.21 & $\mathrm{sf}(+)$ & Al-Salem (1993) \\
serie H & 0.13 & $\mathrm{sf}(\nabla)$ & Janssen and Ribberink(1996) \\
serie C & 0.21 & $\mathrm{sf}(\square)$ & Koelewijn and Ribberink (1994) \\
WI & 0.18 & $\mathrm{r}(\mathbf{})$ & Watanabe and Isobe (1990) \\
& 0.87 & $\mathrm{r}(\bullet)$ & \\
\hline
\end{tabular}

Tab. 1 - Base de donnés testée (sf - sheet flow; $r$-rides)

Experimental data set considered 


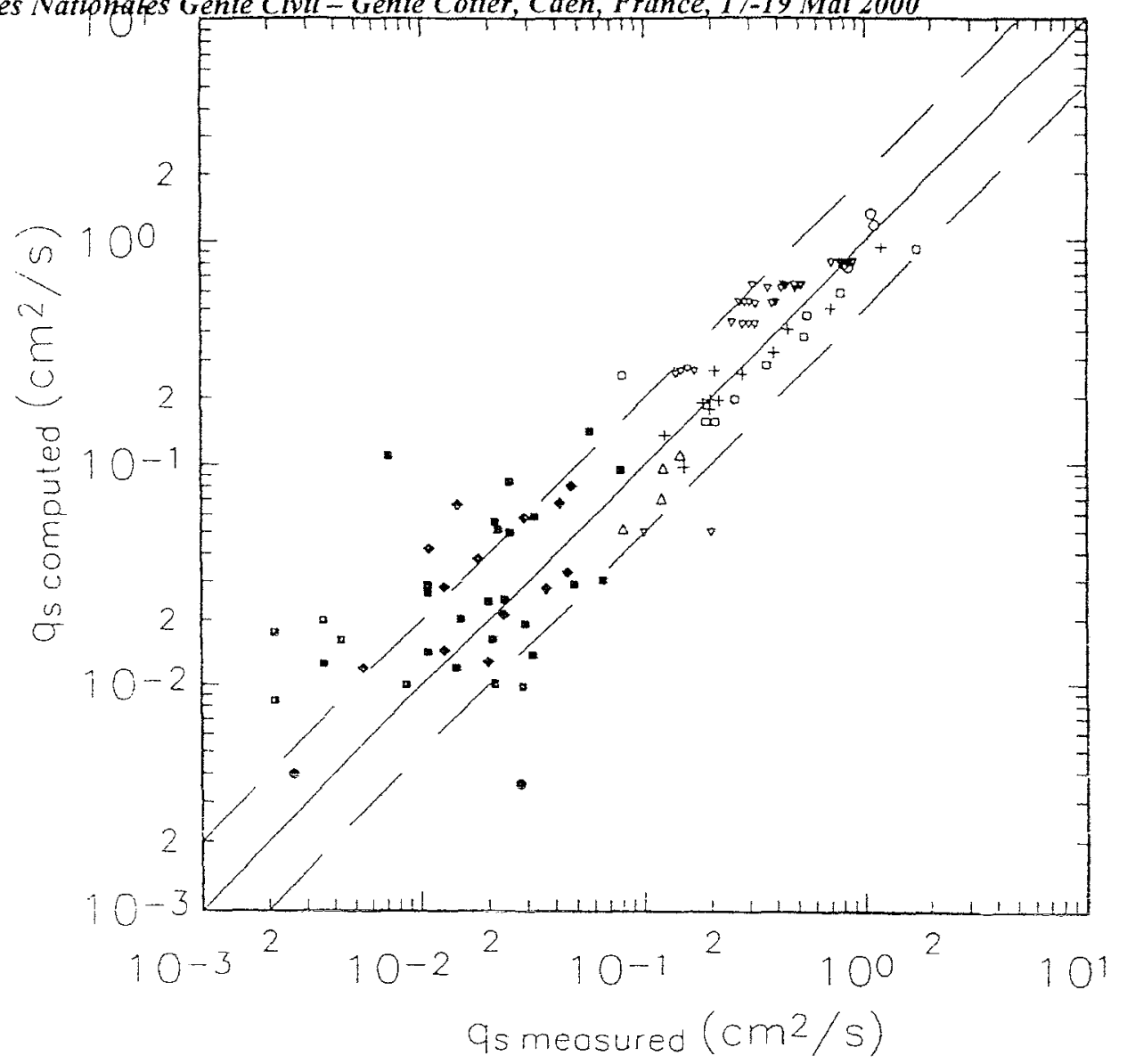

Fig. 4 - Comparaison entre les valeurs calculées et mesurées du débit sédimentaire pour une centaine d'expériences

comparison of the computed and measured net sediment transport rate.

\section{Conclusions}

Nous avons établi pour le débit sédimentaire sous l'action d'une houle régulière en interaction avec un courant, une formule analytique basée sur le model de D-W où nous avons modifié l'expression de $\varpi_{c r}$. Pour une centaine d'expériences testées les résultats obtenus sont dans une rapport inférieur à deux pour plus de $60 \%$ des cas. Pour le cas des houles aléatoires la formulation peut être appliquée, il suffit de préciser les valeurs de $\gamma_{i}$ et $T_{i}$ en fonction des pitramètres du spectre. Il en est de même pour le cas des granulométries étalées.

\section{Remerciements}

Les auteurs remercient la CEE pour avoir financé ce travail dans le cadre du projet SEDMOC (contrat MAS3-CT97-0115).

\section{Bibliographie}

- AI-SALEM A. (1993) - Sediment transport in oscillatory boundary layers under sheet-flow conditions, PhD Thesis, Delft University of Technology. 
- BAILARD J.A. (1981) - An energetic total load sediment transport model for a plane sloping beach, J. Geophysical Res., 86 (C11), pp. 10938-54.

- BYRD P.F., FRIEDMAN M.D., (1954) - Handbook of elliptical Integrals, Springer Verlag, New York.

- DAVIES A. G., SOULSBY R.L., KING H.L. (1988) - A numerical model of the combined wave and current bottom boundary layer, J. Geoph. Res., 93 (Cl), pp.491-508.

- DIBAJNIA M. (1995) - Sheet flow transport formula extended and applied to horizontal plane problems, Coastal Eng. in Japan, JSCE, Vol. 38(2), pp. 179 194.

- DIBAJNIA M., WATANABE A. (1992) - Sheet flow under non-linear waves and currents, Proc. $23^{\text {rd }}$ ICCE, ASCE, pp. 2015-2028.

- DIBAJNIA M., WATANABE A. (1996) - A transport rate formula for mixedsize sands, Proc. $25^{\text {th }}$ ICCE, ASCE, pp. 3791-3804.

- DOHMEN-JANSSEN M. (1999) - Grain size influence on sediment transport in oscillatory sheet flow, PhD Thesis, Delft University of Technology.

- FREDSOE J. (1984) - Turbulent boundary layer in wave-current motion, J. Hydraul. Eng., ASCE, 110, pp.1103-20.

- FREDSOE J., DEIGAARD R. (1992) - Mechanics of coastal sediment transport, World Scientific Publ., Advanced Series ion Coastal Eng., vol 3.

- GRANT W.D., MADSEN O.S. (1979) - Combined wave and current interaction with a rough bottom, J. Geoph. Res., 84 (C4), pp.1797-1808.

- GUIZEN K., TEMPER VILLE A. (1999) - Frottement de fond sous une houle irrégulière linéare, C.R. Acad. Sci. Parist. 327, série II b, pp. 1375-78.

- HORIKAWA K. (1988) - Nearshore dynamics and coasial processes, University of Tokyo Press.

- HUNHN THANH S., TEMPERVILLE A. (1990) - A numerical model of the rough turbulent boundary layer in combined wave and current interaction, Proc: $22^{\text {th }}$ Coustal Eng. Conf., 853-866.

- JANSSEN C. M., RIBERRINK J. (1996) - Influence of grain diameter on sand transport in oscillatory sheet flow, Proc. $25^{\text {th }}$ ICCE, ASCE, pp. 47794792. 
- KATOPODI I., RIBBERINK J.S., RUOL P., KOELEWIJN R., LODAHL C., LONGO S., CROSATO A., WALLACE H. (1994) - Intra-wave sediment transport in an oscillatory flow superimposed on a mean current, Data report H 1684, Part III, Delft Hydraulics.

- KOELEWIJN H., RIBBERINK J.S. (1994) - Seciment transport under sheet flow conditions, Delft University of Technology, Department of Civil Engineering, Delft Hydraulics.

- MYRHAUG D., SLAATTELD O.H. (1990) - A rational approach to wavecurrent friction coefficient for rough. smooth and transitional turbulent flow, Coastal Eng. 14, pp. 25-293.

- NIELSEN P. (1992) - Coastal bottom boundary layers and sediment transport, World Scientific Pub., Advanced Series on Coastal Eng., vol. 4.

- RIBBERINK J., AL-SALEM A. (1904) - Sediment transport in oscillatory boundary layers in cases of rippled bed and sheet flow. J. Geophysical Res., 99 (C6) pp. 12707-12727.

- SLEATH J.F.A. (1984) - Sea bed mechanics, Wiley Interscience.

- SOULSBY R. (1997) - Dynamics of marine sands, Thomas Telford Publications, London.

- VAN RIJN L.C. (1993) - Principles of sediment transport in rivers, estuaries and coastal seas, Aqua Publications, Amsterdam.

- WATANABE A., ISOBE M. (1990) - Sand transport rate under wavecurrent action, Proc. 22 $2^{\text {nd }}$ ICCE, ASCE, pp. 2495-2507. 